\title{
A METODOLOGIA DA TEORIA FUNDAMENTADA NOS DADOS CONSTRUTIVISTA: CONSIDERAÇÕES SOBRE SEU USO NA ÁREA DE ENFERMAGEM EM CUIDADOS COM FERIDAS CRÔNICAS
}

\author{
Idevania G. Costa1, Deborah Tregunno² e Pilar Camargo-Plazas ${ }^{2}$ \\ ${ }^{1}$ Lakehead University, Thunder Bay, ON, Canada. igcosta@lakeheadu.ca \\ ${ }^{2}$ Queen's University, Kingston, ON, Canada. treguno@queensu.ca, mdpc@queensu.ca
}

\begin{abstract}
Resumo: Constructivist Grounded Theory, traduzida para o português como Teoria Fundamentada nos Dados Construtivista (TFDC). Objetivo: descrever nossa experiência com o uso da metodologia da TFDC na área de enfermagem em cuidados com feridas. Métodos: Amostragem teórica e entrevistas semiestruturadas foram realizadas com 30 indivíduos com úlcera de pé diabético, sendo atendidos em uma clínica de feridas no sudeste de Ontário, Canadá, em agosto de 2017. A análise e a interpretação dos conteúdos transcritos foram desenvolvidas de acordo com a proposta metodológica da TFDC informada por Charmaz e utilizou-se o software NVivo 11.4.1 como suporte às interpretações e organização documental. A análise dos dados resultou na teoria: "A Jornada do Paciente rumo ao engajamento no autocuidado do pé diabético". Conclusões: Este estudo permitiu concluir que o método da TFDC é relevante para o desenvolvimento de pesquisas em enfermagem especializada em cuidados com feridas. Devido ao seu rigor analítico e metodológico, podemos entender as várias fases do processo de autocuidado de úlceras de pé diabéticos e o papel central que os pacientes exercem no manejo diário de suas feridas crônicas.
\end{abstract}

Palavras chaves: Teoria Fundamentada em Dados; Autocuidado; Úlcera de Pé Diabético, Fatores Contextuais

THE CONSTRUCTIVIST GROUNDED THEORY METHODOLOGY: CONSIDERATIONS ON ITS USE IN THE AREA OF NURSING IN WOUND CARE

Abstract: Constructivist Grounded Theory (CGT), translated to Portuguese as Teoria Fundamentada nos Dados Construtivista. Goal: to describe our experience with the use of the CGT methodology in the field of nursing in wound care. Methods: Theoretical sampling and semi-structured interviews were conducted with 30 individuals with diabetic foot ulcers, being seen at a wound care clinic in southeastern Ontario, Canada, in August 2017. The analysis and interpretation of the transcribed contents were developed according to the CGT methodological informed by Charmaz and the NVivo 11.4.1 software was used to support the interpretations and data management. The analysis of the data resulted in the theory: "Patients' Journey toward engagement in self-management of diabetic foot ulcer". Conclusions: This study allowed us to conclude that the CGT methodology is relevant to the development of research in nursing specialized in wound care. Due to its analytical and methodological rigor, we could understand the various phases of the engagement in the self-management process of diabetic foot ulcers and the central role that patients play in the daily management of their chronic wounds.

Keywords: Grounded Theory; Self-Management; Diabetic Foot Ulcer, Contextual Factors

\section{INTRODUÇÃO}

Feridas crônicas, como úlceras de pé diabético (UPD), afetam profundamente a vida das pessoas que vivem com elas e resultam em um alto custo, significativo para indivíduos, famílias, 
sistemas de saúde e sociedade, como resultado da deficiência física e diminuição da produtividade. De fato, os resultados indesejados do cuidado com diabetes afetam os indivíduos de forma holística, incluindo áreas físicas, psicológicas e sociais de sua vida e podem resultar em consequências graves e irreversíveis, como a amputação de membros inferiores. Atualmente a úlcera do pé diabético é a principal causa de visitas em unidades de emergência, longas internações hospitalares e altas taxas de mortalidade em pacientes com diabetes ao redor do globo (Skrepnek, Mills, \& Armstrong, 2015). Como poderemos prevenir esses resultados indesejáveis?

Nos dias atuais, há muitos avanços em pesquisas na área de feridas crônicas e novas tecnologias disponíveis que prometem excelentes resultados. No entanto, pouca atenção tem sido dada à importância de envolver os pacientes no autocuidado diário de suas feridas crônicas. Isso pode ser pelo fato de o atual modelo biomédico assistencial ter contribuído para que os pacientes sejam participantes passivos no manejo dos cuidados com suas feridas. Nesse modelo os médicos e demais profissionais de saúde, geralmente, tomam a decisão de tratamento em nome do paciente, sem normalmente envolvê-los no processo de tomada de decisão. Desta forma, esse modelo não é adequado para o manejo de doenças crônicas, necessitando uma mudança de paradigma (Farre \& Rapley, 2017). Mas qual seria a proposta de mudança desse paradigma implícita pela literatura?

No cuidado com pacientes que vivem com doenças crônicas é necessário mudar o papel passivo dos pacientes, perpetuado com modelo biomédico, para um papel mais ativo e participativo, que visa o envolvimento ativo dos mesmos em seus autocuidados (Costa, Camargo-Plazas, Tregunno, 2017). De acordo com as evidências, pessoas que vivem com condições crônicas, mas estão ativamente envolvidas em seus próprios cuidados obtiveram resultados clínicos such as controle da glicemia e melhor qualidade de vida, significativamente melhores (Boger et al., 2015). Outros autores analisaram o custo-efetividade da implementação do autocuidado em pacientes com síndrome metabólica concluíndo que houve diminuição das complicações que levam à hospitalização, dos custos de saúde, e aumento da qualidade de vida (Sakulsupsiri, Sakthong \& Winit-Watjana, 2016).

Pesquisadores e clínicos concordam que os resultados positivos do diabetes são fortemente influenciados pela implementações de autocuidado diários pelos pacientes. Mas, para isso, os 
mesmos devem obter conhecimento sobre sua doença, possuírem auto-confiança, serem capazes e suficientemente responsáveis para cuidarem de si mesmos para evitar sérias consequências pela falta de cuidado (Van de Velde et al., 2019). Quais resultados podem ser obtidos com o envolvimento ativo dos pacientes no autocuidados? Indivíduos que se envolvem ativamente no gerenciamento de seus próprios cuidados são mais aptos a identificarem precocemente sinais e sintomas de infecção, tomarem decisões corretas sobre o que fazer, sobre que medidas implementar para evitar consequências irreversíveis (Riegel, Jaarsma, Lee, \& Stromberg, 2019). Além disso, estarão preparados para identificar suas limitações e, assim, saberão a hora certa de procurar ajuda de profissionais qualificados. Evidências também sugerem que as vozes dos pacientes precisam ser ouvidas para ajudar a entender seus papéis e habilidades no manejo de suas condições crônicas (Barello et al., 2015).

Até o momento, poucos estudos têm focado em compreender a experiência cotidiana dos indivíduos com feridas e como envolvê-los no processo de gerenciamento do autocuidado de sua condição. Além disso, não existia um modelo teórico que descrevesse a experiência dos indivíduos que vivem e lidam com UPD, aplicando a práticas diárias do autocuidado. Poucos estudos exploraram os fatores que influenciam as habilidades dos pacientes em aplicarem o autocuidado com tais úlceras. Atualmente, o que se encontra nos estudos publicados é a adaptação de modelos teóricos de autocuidado de condições crônicas, tais como: diabetes, doença pulmonar crônica, insuficiência cardíaca, entre outros. Esses modelos geralmente propõem uma abordagem quantitativa e estritamente positivista, o que limita dar oportunidade para os pacientes expressaram suas experiência, necessidades e preocupações com sua doenças e o autocuidado das mesmas como descritos em estudos qualitativos (Delea et al., 2015; Palaya, Pearson, \& Nash, 2018). Assim, o propósito deste artigo é descrever nossa experiência com o uso da metodologia da TFDC na área de enfermagem em cuidados com feridas.

\section{METODOLOGIA}

Com o intuito de entender como ocorre o processo de envolvimento dos pacientes no autocuidado diário de feridas complexas tais como UPD, decidimos por usar a abordagem construtivista por ser um método que nos permite adotar uma postura profundamente reflexiva chamada autoconsciência metodológica. Essa abordagem nos leva a examinar os dados, ações 
e análises emergentes e permite um diálogo interativo entre o pesquisador e os participantes da pesquisa (Charmaz, 2017). Esse método nos auxiliou no insight sobre conceitos, significados, motivações e ações em torno do objeto de pesquisa. Na TFDC, os pesquisadores estão em constante envolvimento na construção e interpretação dos dados, enfatizando contextos sociais, interação, compartilhamento de pontos de vista e de suas compreensões (Charmaz, 2017). Além disso, os teóricos construtivistas, buscam uma compreensão abstrata do objeto de estudo e veem suas análises localizadas no tempo, no local e na situação da investigação (Charmaz, 2014).

Neste estudo, também utilizamos o interacionismo simbólico que é considerado o marco filosófico da TFDC. Este assume que a interação é inerentemente dinâmica e interpretativa e, portanto, aborda como as pessoas criam, interpretam, endossam e alteram significados e ações em suas vidas (Charmaz, 2014). No interacionismo simbólico, "os seres humanos são vistos como participantes ativos e construtores do mundo em que vivem e interagem" (MacDonald, 2001, p. 117). Esses conceitos foram particularmente relevantes para este estudo, no qual pretendemos entender os significados e as interpretações que os indivíduos com UPD dão à sua experiência de conviver e manejar suas úlceras no cotidiano.

\section{COLETA E GERENCIAMENTO DOS DADOS}

Em estudos que usam a TFDC, recomenda-se fortemente que o pesquisador aborde a coleta e a análise de dados simultaneamente e de forma não linear, o que permite que a coleta de dados informe a análise e se concentre na construção da teoria (Charmaz, 2014). Assim, a coleta de dados envolveu uma amostragem inicial e teórica e o uso de várias fontes, como entrevistas, observações, anotações de campo e diário do pesquisador (Charmaz, 2014).

Os dados foram coletados somente após aprovação pelo Comitê de Ética em Pesquisa com seres humanos da Queen's University, Kingston, Ontário, Canadá assinatura do termo de consentimento informado pelos participantes. Garantimos o anonimato dos participantes usando pseudônimos, escolhidos pelos mesmos, em vez dos seus nomes reais. 


\subsection{Fontes de coleta de dados}

\subsubsection{Guia intensivo de entrevista semiestruturado}

O guia intensivo de entrevista semiestruturado, foi desenvolvido com base na literatura sobre autocuidado e manejo de UPD, na experiência de um dos pesquisadores como enfermeira de tratamento de feridas (da primeira autora) e também informado pela TFDC de Charmaz (2014). Assim, desenvolvemos a primeira versão do guia que foi usada com os cinco primeiros participantes. Após essa experiência, analisamos as repostas e obtivemos insights sobre diferentes abordagens para entender o processo de gerenciamento de autocuidado para essa população e decidimos reformular algumas perguntas. Na segunda versão do guia de entrevistas, decidimos por organizar as perguntas por tópico para evitar repetições. Este novo guia de entrevistas também ofereceu melhor direção, confiança, foco e clareza para que a entrevistadora explorasse o processo de envolvimento do paciente no autocuidado da UPD. Abaixo, fornecemos alguns exemplos das perguntas da entrevista usadas na segunda versão da entrevista, com algumas perguntas investigatórias:

Quadro 1. Exemplos das perguntas da entrevista intensiva semiestruturada

\begin{tabular}{|l|l|}
\hline \multicolumn{1}{|c|}{ Perguntas Centrais } & \multicolumn{1}{|c|}{ Perguntas investigatórias } \\
\hline $\begin{array}{l}\text { Conte-me sobre quando você foi diagnosticado com } \\
\text { diabetes. }\end{array}$ & Inclua todos os detalhes que você lembrar \\
\hline $\begin{array}{l}\text { Como você se cuidou desde que descobriu o seu } \\
\text { diabetes? }\end{array}$ & $\begin{array}{l}\text { Alguma coisa mudou? Onde você busca as respostas } \\
\text { para suas dúvidas? }\end{array}$ \\
\hline $\begin{array}{l}\text { Que tipo de apoio você conseguiu para ajudá-lo a cuidar } \\
\text { de si mesmo desde que foi diagnosticado com diabetes? }\end{array}$ & $\begin{array}{l}\text { Quem ajuda você? De que maneira? O que significa ter } \\
\text { ajuda? }\end{array}$ \\
\hline $\begin{array}{l}\text { Conte-me sobre a primeira vez que você descobriu que } \\
\text { tinha uma ferida no seu pé. }\end{array}$ & Como você reagiu? O que passou pela sua cabeça? \\
\hline Onde você foi primeiro para obter ajuda com sua ferida? & O que você disse sobre como cuidar de sua ferida? \\
\hline
\end{tabular}

\subsubsection{Anotações de campo}

As anotações de campo permitiram ter um lugar para documentar as impressões sobre as experiências dos participantes e reações da entrevistadora (Strauss \& Corbin, 2015). As anotações de campo ajudaram a entrevistadora a documentar uma descrição importante de dados, como data, hora, ações, comportamentos, ou se os participantes usavam algum tipo específico de sapatos ou botas de prevenção de pressão na sola do pé afetado, bem como conversas paralelas entre o participante e o entrevistador. Selecionamos, a seguir, um bom exemplo da anotação de campo feita pelo entrevistador (primeiro autor): 
Anotação de Campo (26 de maio de 2017)

Shelby (nome fictício) estava me esperando na sala de espera da clínica de tratamento de feridas. Ela estava assistindo TV quando me aproximei e a convidei para entrar na sala privada para a entrevista. Ela usava um dispositivo móvel de redução de pressão no pé afetado (esquerdo) e uma sandália no pé não afetado (direito). Ela sorriu sutilmente quando me aproximei dela e, à minha primeira impressão, foi de que ela não parecia interessada em falar sobre sua experiência de ter e cuidar de seu diabetes e ulceração. Shelby, uma participante do sexo feminino de 52 anos, começou a ter sinais de complicações do diabetes após quinze anos do diagnóstico de diabetes tipo 2. Ela compartilhou: "Eu perdi minha visão e não podia ver propriamente. Fui ao hospital, e então me diagnosticaram com diabetes. Meu açúcar no sangue estava 29 na época. Shelby estava frustrada porque na época ela não tinha um médico de família para prescrever os remédios que ela precisava e levou dois anos e meio para conseguir um. Shelby revelou que havia saído dos trilhos com sua diabetes por muitas vezes e por isso não se considera uma "boa diabética "porque não leva à sério sua dieta, não faz exercícios físicos e não consegue parar de fumar. A primeira vez que ela detectou a úlcera no pé, ela tentou tratá-la por conta própria aplicando remédios que ela encontrou na prateleira das farmácias. No entanto, a úlcera piorou e ela procurou uma clínica de emergência, onde o médico receitou um antibiótico que também não funcionou bem. Ela continuou com infecção na UPD por mais de dois meses sem saber o que fazer e o que era aquilo.

\subsubsection{Diário do pesquisador}

Ao longo do processo de estudo, o diário do pesquisador ajudou a detalhar o pensamento sobre a área de pesquisa e como ela pode influenciar a análise dos dados (Strauss \& Corbin, 2015). Este diário serviu como um local para escrever ideias e interpretar as reações dos participantes ao convite para participar da pesquisa ou suas reações a algumas perguntas (por exemplo, apoio e recursos necessários para cuidar do diabetes e UPD). Também nos ajudou a entender que os indivíduos com UPD, às vezes, enfrentam muitos problemas pessoais e de saúde que podem afetar seu envolvimento no autocuidado. Um exemplo desse diário reflexivo está descrito abaixo:

Diário do pesquisador (26 de maio de 2017)

Esta é minha reflexão pessoal depois de entrevistar a senhora Shelby. Depois de uma semana tentando marcar uma entrevista com ela, finalmente tive a chance de encontrá-la na sala de tratamento de feridas enquanto ela estava sendo atendida por sua enfermeira habitual. Minha primeira impressão depois de conversar com ela foi que ela tinha medo de falar sobre o assunto, portanto, evitava 0 contato visual comigo na sala de tratamento de feridas. No início da entrevista, ela novamente evitou o contato visual. Aprendi com ela que ela tinha medo de ser julgada por não estar sempre seguindo as orientações de seu tratamento de feridas. Ela compartilhou que havia sido questionada muito sobre ser fumante e ter uma ulceração, mas que era um desafio para ela parar de fumar. Minha impressão foi de que ela passou por muitas situações difíceis em sua vida, como por exemplo,depressão, a qual interferiu no seu desejo de implementar o tratamento para sua UPD e o controle do diabetes. A experiência de Shelby de ter muitas recorrências do UPD me ensinou que é fácil perder a esperança. Esta entrevista contribuiu muito para meu entendimento amplo sobre o fenômeno em estudo. Percebi que embora os pacientes desempenhem um papel importante no autocuidado e na cicatrização de suas feridas, seu sucesso depende de como esses foram afetados por seu contexto social enquanto vivem com tal problema de saúde e como esse problema também afetou esse ser social. 


\section{2 Organização e gerenciamento dos dados}

As informações obtidas nas entrevistas foram inseridas no software de gerenciamento de dados qualitativo $\mathrm{N}-V_{i v o}{ }^{\odot}$ (Versão 11.4.1). Esse software foi essencial para auxiliar no gerenciamento e análise de todos os dados. Ao todo foram entrevistados 30 participantes e a transcrição de suas entrevistas originou um total de 732 páginas que foram inseridas no NVivo 11.4.1.

As entrevistas realizadas por telefone para validar e esclarecer os dados originaram outras 87 páginas que também foram inseridas no software com o auxílio de um computador. O número de páginas de cada entrevista, após transcrita, variou de 15 a 56 e a duração das entrevistas variou de 36 minutos e 41 segundos à 1 hora e 42 minutos. O NVivo foi usado para ajudar com os dois tipos de codificações utilizadas na TFDC - codificação inicial ou aberta e seletiva ou focada. Os códigos foram organizados em Nodes no NVivo 11.4.1.

\section{ANÁLISE DE DADOS}

\subsection{Procedimentos de codificação do dados}

O processo de codificação da TFDC envolve pelo menos as duas codificações hierárquicas a seguir: 1) codificação aberta (inicial) linha a linha ou ideia da ideia e 2) uma codificação focalizada (seletiva e conceitual) (Charmaz, 2014) como descrito a seguir.

\subsubsection{Codificação aberta}

A codificação aberta é o primeiro passo na análise da TFDC e ajuda o pesquisador a continuar aberto aos dados e a ver distinções nos mesmos. Essa forma de codificação requer a análise de cada uma das entrevistas transcritas, bem como de suas histórias, linha por linha. Ao codificar os dados da entrevista em profundidade, o pesquisador examina de perto o que os participantes dizem e, provavelmente, enfrenta (Charmaz, 2014). Assim, neste estudo, a codificação aberta nos ajudou a identificar preocupações implícitas e declarações explícitas. O envolvimento no processo de codificação aberta, a partir da primeira entrevista, nos ajudou a manter o foco durante toda a coleta e análise de dados. Essa codificação aberta foi muito útil para identificar a percepção dos participantes de não controlar muito bem o diabetes, que foi 
codificado com as próprias palavras dos participantes de "ser um diabético ruim", de forma a manter os códigos o mais próximos possível dos dados. Os trechos abaixo indicam texto usado nesta codificação. O código "ser um diabético ruim" foi posteriormente elevado à um conceito.

O que significa "Ser um diabético ruim" para os participantes:

Eu sou um diabético ruim. (Júnior).

Eu não sou um diabético muito bom, com certeza. (Shelby)

Eu costumava comer muita fast food ... muita Pepsi. (Kobe)

Eu não faço muito exercício. (Charlette)

Eu fumo dois maços de cigarro por dia. (Júnior)

Comecei a beber álcool novamente. (Sam)

Após seguir as estratégias de codificação aberta, percebemos estar imersas no processo de entender as experiências dos participantes de viver e cuidar de suas UPD.

\subsubsection{Codificação focalizada}

A codificação focalizada foi usada para sintetizar e explicar segmentos maiores de dados. Esse tipo de codificação é mais direcionada, focada e conceitual do que o processo de codificação aberta (Charmaz, 2014). Através do processo de comparação constante, os códigos iniciais foram comparados e alguns deles foram unificados de forma a facilitar sua descrição. O método de comparação constante de código com código, depoimento com depoimento e assim por diante nos permitiu refinar o código de "ser um diabético ruim" e elevá-lo à um conceito da categoria intitulada: "Percepção da doença" que se tornou a Fase 1 da teoria desenvolvida neste estudo.

\subsubsection{Análise comparativa constante}

A análise comparativa constante é um método chave na análise de dados da TFDC (Charmaz, 2014). Esse método nos ajudou a entender o processo incorporado na jornada dos participantes rumo ao envolvimento no autocuidado de suas UPD, em vez de impor ideias preconcebidas relacionadas ao processo de envolvimento dos participantes no autocuidado de 
suas UPD. Além disso, esse método indutivo de análise, nos ajudou a estender significados e experiências e a desenvolver conceitos a partir dos dados enquanto os analisavam (Charmaz, 2014).

\subsubsection{Redação de memorandos e categorias conceituais}

É neste momento que os pesquisadores param e analisam suas ideias sobre os códigos e categorias emergentes (Charmaz, 2014). Portanto, é essencial escrever o memorando imediatamente após a leitura e codificação das entrevistas. Assim, anotações sobre o que o pesquisador estava pensando, as ideias que surgiam quando lia as transcrições dos entrevistas, foram anotadas na fonte denominada Memo do software NVivo. A redação de memorandos também foi muito útil para criar um espaço interativo onde o pesquisador pudesse manter uma conversa consigo mesmo sobre dados, códigos, ideias e intuições e liderar o desenvolvimento da análise e construção da teoria, como afirma Charmaz. Um exemplo da escrita de memorando é fornecido abaixo:

Memorando (July 31, 2017)

Depois de codificar algumas entrevistas de participantes como Junior, Butch e Toby, que enfrentaram muitas consequências do diabetes, percebi que eles veem a doença como uma "sentença de morte" e pareciam preparados para perder a vida à qualquer momento. Eles também admitiram que não eram um "bom diabético" porque não seguiram uma dieta rigorosa ou manejaram seu diabetes como Ihes foi dito. Outra coisa comum entre eles era que os mesmos não levavam isso a sério desde o início, e que a falta de conhecimento e apoio no autocuidado os levou a não conseguir acompanhá-lo diariamente. Agora eles pareciam aceitar que morreriam devido às várias consequências do diabetes e estão preparando seus entes queridos para aceitarem esse resultado também. Por outro lado, aqueles que apresentavam menos consequência estavam cientes dos riscos para sua vida, mas ainda sentiam que podiam controlar seu diabetes e viver bem e por muito tempo com a doença.

\section{SATURAÇÃO TEÓRICA}

A TFDC difere no uso desse termo comum de saturação de dados. Para este estudo, a saturação dos dados foi alcançada quando duas situações ocorreram. Primeiro, as categorias teóricas eram robustas e cheias de dados (Charmaz, 2017). Isso ocorreu quando não foram obtidas novas propriedades para a categoria principal "A jornada rumo ao envolvimento no autocuidado de UPD”. A saturação teórica foi alinhada com a técnica de amostragem teórica utilizada durante a pesquisa para orientar a necessidade de entrevistas adicionais ou as entrevistas com os mesmos participantes. Segundo, a saturação teórica foi alcançada quando 
os dados coletados foram responsáveis desenvolver as relações teóricas e o entendimento da trajetória dos participantes rumo ao processo de engajamento no autocuidado de UPD. Portanto, neste estudo, a saturação dos dados ocorreu quando novos dados não alteraram os conceitos já descobertos. Em vez disso, novos dados continuaram apenas a confirmar e promover os conceitos já conhecidos.

\section{CLASSIFICAÇÃO TEÓRICA}

A classificação teórica é a última etapa do processo de pesquisa para escrever uma teoria com a metodologia da TFDC. Nesta fase, a classificação, diagramação e integração de memorandos se tornam um processo inter-relacionado. Esse processo nos ajudou a encontrar a direção rumo à construção da teoria. A classificação dos memorandos serviu para expor a teoria emergente e forneceu meios para criar e refinar os vínculos teóricos (Charmaz, 2014). A diagramação ofereceu imagens concretas das nossas ideias e nos ajudou a visualizar e mostrar a representação das propriedades da teoria, seus relacionamentos e como cada propriedade se encaixava. Tentamos várias estratégias de classificação diferentes, desenhamos vários diagramas e comparamos categorias com categorias várias vezes até ter uma boa representação visual de seus links e encaixes. Integrando os dados e desenvolvendo a teoria substantiva

\section{SENSIBILIDADE TEÓRICA E REFLEXIVIDADE}

A sensibilidade teórica refere-se ao atributo de ter os insights necessários para dar significado conceitual aos dados. Também reflete a capacidade do investigador de usar experiências pessoais e profissionais para descobrir, entender e interpretar um contexto e dados de pesquisa de uma maneira que permita ao pesquisador explicar o potencial das informações para 0 desenvolvimento da teoria (Charmaz, 2014). O autor afirma que os conceitos de sensibilização permitem ao pesquisador expandir a percepção e fornecer um ponto de partida para a construção da análise.

Estudos preocupados com a geração de teoria podem exigir conceitos sensibilizantes, mas sem hipóteses (Blaikie, 2017). Em vez de começar com uma hipótese para analisar os dados, a pesquisa qualitativa utiliza o processo indutivo, que significa extrair padrões, conceitos e categorias dos dados, em vez de serem impostos antes da coleta de dados (Bradshaw, 
Atkinson, \& Doody, 2017). Por exemplo, quando comecei a coletar e analisar meus dados de pesquisa, precisava voltar e refletir constantemente sobre minha experiência pessoal e profissional, usando um diário ou diário reflexivo. Isso permitiu uma maior compreensão do que os dados estavam me dizendo em comparação com o que sei da minha experiência sobre a situação.

\section{CONCLUSÕES}

Nosso intuito foi descrever nossa experiência com o uso da TFDC na área de enfermagem em cuidados com feridas. Confirmamos que o uso dessa metodologia nos ajudou a desenvolver a primeira teoria que explica o processo de envolvimento do paciente no autocuidado de UPD, bem como os fatores contextuais que influenciaram esse processo (em processo de publicação). O processo iterativo utilizado neste estudo se deu de forma complexa e abstrata, conforme sugerido por Charmaz. Ao fornecer uma explicação detalhada das etapas adotadas para desenvolver este estudo, essa seção metodológica demonstra a complexidade das atividade de pesquisa e suporta a validade dos resultados.

O desenho da pesquisa foi influenciado por uma abordagem teórica fundamentada no interacionismo simbólico e no construtivismo social. As fontes de coleta de dados incluiu entrevista semiestruturada, anotações de campo e diário do pesquisador. As entrevistas ocorreram até que a saturação teórica ocorresse, um procedimento tradicional das TFDC. Enquanto todos os participantes estavam dispostos a compartilhar suas experiências, seu foco de interesse variou de acordo com os tópicos abordados. Por exemplo, enquanto alguns se interessaram em fornecer detalhes sobre sua experiência em cuidar de suas feridas, outros se concentraram principalmente em suas lutas pessoais ao navegar no mundo social com diabetes e UPD ou no impacto da úlcera em suas vidas.

Durante as entrevistas perguntas investigatórias foram incluídas para ajudar a obter uma descrição aprofundada sobre as reações, ações, motivações e significados que os participantes davam à suas experiências de viver e cuidar de suas UPD diariamente. As anotações de campo e o diário reflexivo forneceram fontes extras de dados onde estão descritas as ações, atitudes e comportamentos dos participantes observados durante a entrevista. A TFDC forneceu métodos flexíveis para guiar o processo analítico de desenvolvimento dessa teoria. 


\section{REFERÊNCIAS}

Barello, S., Graffigna, G., Vegni, E., Savarese, M., Lombardi, F., \& Bosio, A. C. (2015). ‘Engage me in taking care of my heart': A grounded theory study on patient-cardiologist relationship in the hospital management of heart failure. BMJ Open, 5(3), 1-11.

Blaikie, N. W. H. (2017). Designing social research: The logic of anticipation. (3 ${ }^{\text {rd }}$ Ed.) Cambridge, UK: Polity.

Boger, E., Ellis, J., Latter, S., Foster, C., Kennedy, A., Jones, F., . . (2015). Demain, S. Self-Management and Self-Management Support Outcomes: A Systematic Review and Mixed Research Synthesis of Stakeholder Views. PloS one, 10(7), 1932-6203 (Electronic).

Bradshaw, C., Atkinson, S., \& Doody, O. (2017). Employing a Qualitative Description Approach in Health Care Research. Glob Qual Nurs Res, 4, 1-8. doi:10.1177/2333393617742282

Charmaz, K. (2014). Constucting Grounded Theory (2nd ed.). Washington, D.C: Sage.

Charmaz, K. (2017). The Power of Constructivist Grounded Theory for Critical Inquiry. Qualitative Inquiry, 23(1), 34-45. doi:10.1177/1077800416657105

Costa, I. G., Camargo-Plazas. M. P., \& Tregunno. D. (2017). Re-thinking self-care management for individuals with diabetic foot ulcers. Wounds International, 8(2); 10-14.

Delea, S., Buckley, C., Hanrahan, A., McGreal, G., Desmond, D., \& McHugh, S. (2015). Management of diabetic foot disease and amputation in the Irish health system: a qualitative study of patients' attitudes and experiences with health services. BMC health services research, 15(1), 251. doi:10.1186/s12913-015-0926-9

Farre, A., \& Rapley, T. (2017). The new old (and old new) medical model: four decades navigating the biomedical and psychosocial understandings of health and illness. Healthcare (Basel, Switzerland), 5(4), 88.

MacDonald, M. (2001). Finding a critical perspective in grounded theory. In R. Schreiber \& P. N. Sterm (Eds.), Using Grounded Theory in Nursing (pp. 113-158). New York: Springer Publishing Company.

Palaya, J., Pearson, S., \& Nash, T. (2018). Perception of social support in individuals living with a diabetic foot: A qualitative study. Diabetes Res Clin Pract, 146, 1872-8227

Riegel, B., Jaarsma, T., Lee, C. S., \& Stromberg, A. (2019). Integrating symptoms into the middle-range theory of self-care of chronic illness. ANS Adv Nurs Sci., 42(3), 1550-5014.

Sakulsupsiri, A., Sakthong, P., \& Winit-Watjana, W. (2016). Cost-Effectiveness Analysis of the Self-Management Program for Thai Patients with Metabolic Syndrome. Value in Health Regional Issues, 9, 28-35

Skrepnek, G. H., Mills, J. L., Sr., \& Armstrong, D. G. (2015). A diabetic emergency one million feet long: Disparities and burdens of illness among diabetic foot ulcer cases within emergency departments in the United States, 2006-2010. PLoS One, 10(8), 1-15. doi:10.1371/journal.pone.0134914

Strauss, A., \& Corbin, J. (2015). Basics of qualitative research: Techniques and procedures for developing grounded theory $\left(4^{\text {th }}\right.$ ed.). Thousand Oaks, CA: Sage.

Van de Velde, D., De Zutter, F., Satink, T., Costa, U., Janquart, S., Senn, D., \& De Vriendt, P. (2019). Delineating the concept of self-management in chronic conditions: a concept analysis. BMJ open, 9(7), e027775. 